\title{
1 Spectral detection of stress-related pigments in salt-lake succulent halophytic shrubs
}

2

3 Victoria A. Marchesini ${ }^{1,2^{*}}$, Juan P. Guerschman ${ }^{3}$, Ralf M. Schweiggert ${ }^{4}$, Timothy D. Colmer ${ }^{1}$ \& Erik. J. $4 \quad$ Veneklaas $^{1}$

5

$6{ }^{1}$ School of Plant Biology, The University of Western Australia, 35 Stirling Highway,

7 Crawley WA 6009, Australia

$8 \quad{ }^{2}$ GEA-IMASL/CONICET, Ejército de los Andes 950, San Luis, Argentina.

$9 \quad{ }^{3}$ CSIRO Land and Water, GPO Box 1666, Canberra, ACT 2601, Australia

$10{ }^{4}$ Institute of Food Science and Biotechnology, University of Hohenheim, 70599 Stuttgart, Germany.

*Corresponding author: victoria.marchesini@gmail.com

13 Abstract

14 The spectral detection of vegetation pigment concentrations has a high potential value, but it is still underdeveloped, especially for pigments other than chlorophylls. In this study, the seasonal pigment dynamics of two Tecticornia species (samphires; halophytic shrubs) from north-western Australia were correlated with spectral indices that best document the pigment changes over time. Pigment dynamics were assessed by analysing betacyanin, chlorophyll and carotenoid concentrations at plant level and by measuring reflectance at contrasting seasonal dates. Plant reflectance was used to define a new reflectance index that was most sensitive to the seasonal shifts in Tecticornia pigment concentrations.

21 The two Tecticornia species turned from green to red-pinkish for the period March-August 2012 when 
betacyanins increased almost nine times in both species. Chlorophyll levels showed the opposite pattern to that of betacyanins, whereas carotenoid levels were relatively stable. Normalised difference indices correlated well with betacyanin $(r=0.805$, using bands at 600 and $620 \mathrm{~nm})$ and chlorophyll $(\mathrm{r}=0.809$, using bands at 737 and $726 \mathrm{~nm}$ ). Using knowledge of chlorophyll concentrations slightly improved the ability of the spectral index to predict betacyanin concentration ( $\mathrm{r}=0.822$ at bands 606 and $620 \mathrm{~nm}$, in the case of chemically determined chlorophyll, $r=0.809$ when using remotely sensed chlorophyll). Our results suggest that this new spectral index can reliably detect changes in betacyanin concentrations in vegetation, with potential applications in ecological studies and environmental impact monitoring.

Keywords: inland marshes, betacyanin, chlorophyll, halophytes, spectral indices, plant reflectance, samphire, Tecticornia indica, Tecticornia auriculata.

\section{Introduction}

Plant pigments and variations in their concentrations can be correlated to the state of vegetation during seasonal changes, such as the decline of chlorophylls and the increase of carotenoids and anthocyanins during autumn (Archetti 2009). Pigments in plants can be measured directly by chemical and instrumental analyses or indirectly by spectrometry, since the reflectance in the visible region of the spectrum varies according to the composition and the concentration of many components (Ustin et al. 2004, Ustin et al. 2009). Research on the spectral detection of plant pigments has led to the development of several spectral indices, but most investigations have focussed on chlorophylls due to their importance for photosynthesis (Gamon and Surfus 1999, Gitelson et al. 2009, Peñuelas et al. 2011). There have been fewer spectral studies of other plant pigments, perhaps because their overlapping absorbance with chlorophylls makes it difficult to define indices that are able to provide accurate estimates of non-chlorophyll pigments (Sims and Gamon 2002). 
46 An advantage of using spectrometry to detect pigments is that vegetation condition can be evaluated at

47 large scales (e.g. by satellites), and it is relatively fast compared to traditional methods (Kerr and

Ostrovsky. 2003). Additionally, spectrometry via remote sensing can be especially useful in areas where human intervention is difficult due to their fragility and/or inaccessibility, such as deserts or flooded areas (Shuman and Ambrose 2003). Wetlands and salt marshes are complex ecosystems that show particular interactions between structure (soil and vegetation cover, species composition), and function (nutrient cycling, primary productivity) (Kelly et al. 2011). In seasonal wetlands, the occurrence of flooding events alternating with drought periods controls plant distribution, with environmental gradients resulting in unique microhabitats with highly specialised species. Due to difficult accessibility and complex bio-physical dynamics, wetlands and marshes pose big challenges for monitoring using traditional techniques such as vegetation surveys and other sampling methods (Zomer et al. 2009).

In Western Australia, the halophytic endemic Tecticornia genus (formerly Halosarcia) of the Amaranthaceae family (formerly Chenopodiaceae) is dominant in saline inland areas (Wilson 1980, Shepherd and Van Leeuwen 2010). Australia is a centre of diversity for this particular group (Shepherd and Wilson 2007). Many species grow in ephemeral inland salt lakes that are subject to periodic changes in salinity, waterlogging and drought (Shepherd and Van Leeuwen 2010, English and Colmer 2011). It has been observed that Tecticornia species, especially those occurring in salt marshes, change colouration under unfavourable conditions (e.g. drought or waterlogging) from green to red-pinkish tones (Datson 2002). Changes in colouration seem to be the result of chlorophyll degradation, as well as increases in other pigments like betalains, which are recognized for their role as photoprotective pigments (Stintzing and Carle 2004, Brockington et al. 2011). Betalains are a group of pigments commonly found in the Amaranthaceae family (Strack et al. 2003). Two types of betalains are known: the yellow and orange betaxanthins and the red-violet betacyanins, with absorption maxima at 460-480 
and $540 \mathrm{~nm}$, respectively (Stintzing and Carle 2004). Although the biological functions of betalains are not well understood, their accumulation has been associated with protection against UV radiation (Ibdah et al. 2002) and can be induced by abiotic stresses such as salinity (Hayakawa and Agarie 2010, Zhao et al. 2010, Rabhi et al. 2012).

Despite the importance of Tecticornia species as keystone species dominating in some areas of Australia (Wilson 1980, Shepherd and Wilson 2007), the dynamics and the nature of their pigments have not been spectrally evaluated, and the potential application of spectral signals for the assessment of the condition of these ecosystems is novel. In addition, large areas occupied by Tecticornia have experienced intensive land use changes in the last past decades, such as groundwater extraction and flow diversion for mining, and secondary salinity in agricultural landscapes (Timms 2005). Thus, the evaluation of plant condition and the changes associated with seasons and stress is of importance for conservation and land management.

In this study we assessed the seasonal changes in colouration and the spectral response to these changes for two Tecticornia species from the Fortescue Marsh, the largest inland marsh of the Pilbara region in Western Australia. After elucidating the yet unknown Tecticornia betacyanin pattern by HPLC-DAD$\mathrm{MS}^{\mathrm{n}}$, our main goal was to identify a spectral index that best reflects differences in pigment concentrations. Our hypothesis was that due to seasonal changes, pigment concentration would vary during the year and that the changes in light reflectance could be spectrally detected. To test this hypothesis, we quantified plant pigment dynamics by sampling and analysing plant tissue pigment concentrations (betalains, chlorophylls and carotenoids) and by measuring light reflectance at plant level 
92

93

94 2. Methods

95

96

97

98

\subsection{Study area}

across seasons. Plant reflectance was subsequently used to define a new reflectance index that was most sensitive to the seasonal shifts in Tecticornia pigment concentrations.

Field work was performed in an area on the northern shore of Fortescue Marsh, a basin in the Fortescue River catchment located $120 \mathrm{~km}$ north of Newman, Pilbara region, Western Australia $\left(22^{\circ} 22.9^{\prime} \mathrm{S}, 119^{\circ}\right.$ 20.1' E). The Fortescue Marsh has been recognized for its conservation value and ecosystem services including habitat for several endemic species (Shepherd and Van Leeuwen 2010). The marsh is subjected to episodic monsoonal summer floods alternating with prolonged drought during winter, when soils dry and salinity increases considerably (Skrzypek et al. 2013). Rains mainly occur from October to March with an average of approximately $400 \mathrm{~mm}$ per year. A strong seasonality is also observed in air temperature and solar radiation (Fig. 1). Prior to the study period, heavy rain fell in January and March; during the study period, rain was negligible and conditions became progressively drier but with lower temperature and lower radiation load. Two Tecticornia species were studied: Tecticornia indica subsp. bidens (Nees) K. A. Sheph. and Paul G. Wilson, a C4 species occurring towards the outer margins of the marsh, and the C3 species T. auriculata (K. A. Sheph. and Paul G. Wilson) distributed from the $T$. indica habitat to several hundred meters into the saline marsh at slightly lower elevations (for photographs see Fig S1). Tecticornia species are the dominant perennial shrubs in the area representing almost $90 \%$ of the total plant cover (Moir-Barnetson 2015) 


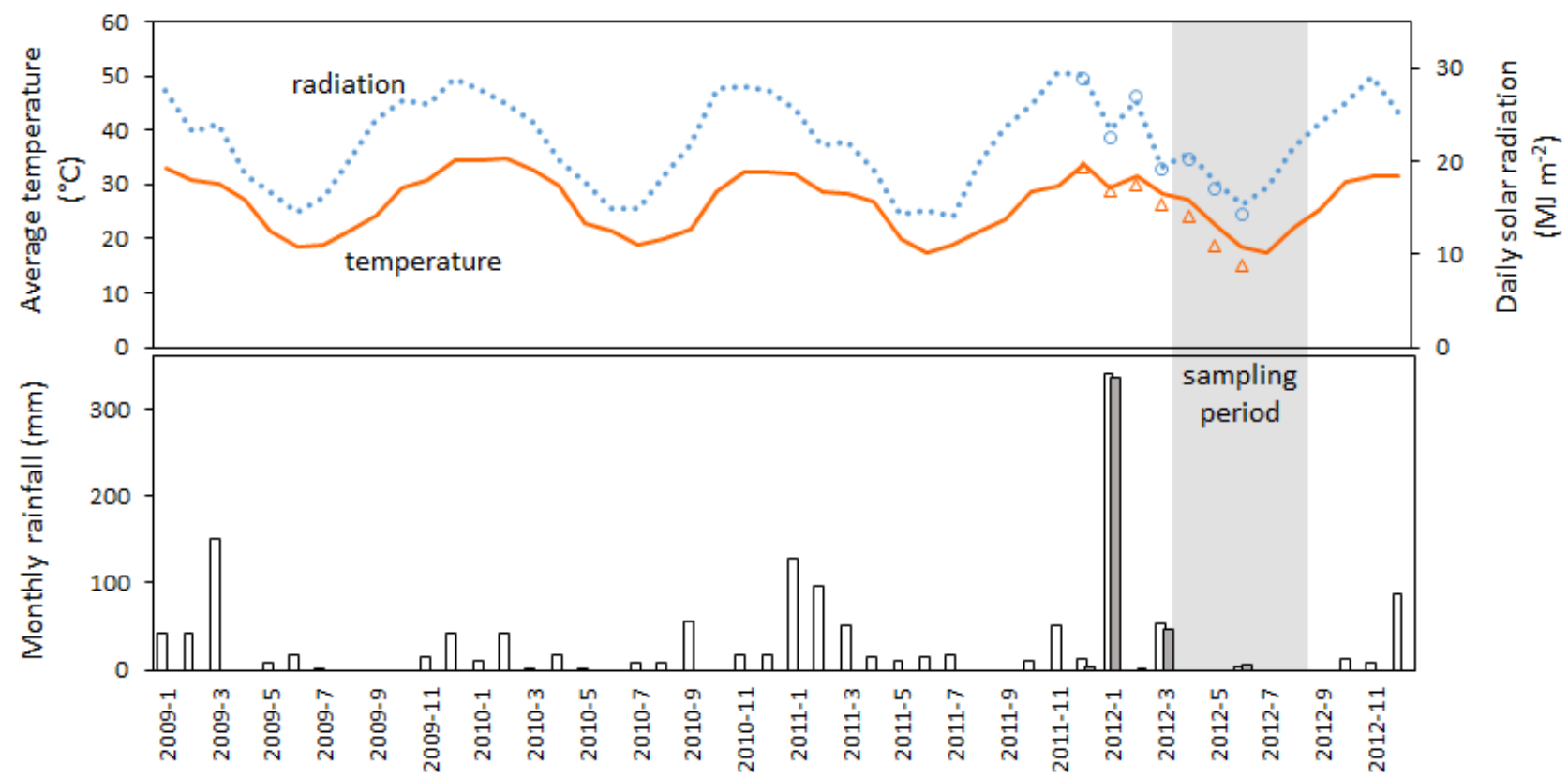

112 Figure 1. Weather conditions in the study area. The top panel shows monthly average air temperature

$113\left({ }^{\circ} \mathrm{C}\right.$; solid line for nearby permanent weather station, triangles for measurements at study site $)$ and

114 daily total solar radiation $\left(\mathrm{MJ} \mathrm{m}^{-2}\right.$; dotted line for nearby permanent weather station, circles for

115 measurements at study site). The bottom panel shows monthly total rainfall (mm; white bars for

116 nearby permanent weather station, grey bars for measurements at study site). The shaded area

117 indicates the period during which spectral and chemical sampling was carried out. The 2009-2011

118 weather data are from stations Marillana (radiation and rainfall; $30 \mathrm{~km} \mathrm{~S}$ of study site) and Wittenoom

119 (temperature; $100 \mathrm{~km}$ W of study site), which were obtained from the Bureau of Meteorology

120 database (bom.gov.au/climate/data).

\subsection{Experimental design}

123 Plant sampling for pigment analysis and spectral measurements was done in three plots per species, with

124 four plants per plot. The size of each plot was $25 \mathrm{~m}^{2}$. Plots were chosen based on a dominance of $90 \%$ 125 of the target species and there was a minimal distance of $100 \mathrm{~m}$ between each plot. Tecticornia plants 
126

127

128

129

130

131

132

133

134

135

136

137

138

139

140

141

142

143

144

145

147

sampled within each species were similar in plant height and crown diameter. Sampling was done in (i) early March 2012 (middle of 'wet' season), (ii) early July 2012 (middle of dry season), and (iii) late August 2012 (late dry season).

\subsection{Pigment analysis}

Representative parts of the succulent plant 'stems' (i.e., reduced and fused leaves) were sampled for pigment analysis and tissue water content. Two large branches (from lowest succulent article to tip) were kept on ice in the dark for up to 6 hours during transport to the laboratory and then snap-frozen in liquid $\mathrm{N} 2$ and subsequently freeze-dried and stored at $-20^{\circ} \mathrm{C}$ to preserve the samples. Betacyanin and chlorophyll were analysed for plants sampled at three strategic times: March, July and August 2012. The reason for choosing these sampling periods was that they represent a transition from hot and moist to cooler but drier and stressed conditions, when the seasonal changes in colouration occur.

Prior to quantitative analyses, the water-soluble pigments present in the Tecticornia sp. were identified by HPLC-DAD-MS ${ }^{\mathrm{n}}$. For this purpose, freeze-dried Tecticornia stems or Amaranthus tricolor leaves (generously provided by Sanjay Nene of the National Chemical Laboratory, Pune, India) were extracted with McIlvaine's buffer (disodium hydrogenphosphate-citric acid buffer, pH 6.5) additionally containing $50 \mathrm{mM}$ L-ascorbate. The aqueous extract was filtered $(0.45 \mu \mathrm{m})$ into amber glass vials and stored at $-20{ }^{\circ} \mathrm{C}$ until HPLC analyses. HPLC-DAD-MS ${ }^{\mathrm{n}}$ analyses were conducted as described previously at Schweiggert et al. (2009), except for the different column temperature $\left(25{ }^{\circ} \mathrm{C}\right)$ and a different elution gradient, being based on $1 \% \mathrm{v} / \mathrm{v}$ formic acid in water (solvent A) and methanol (solvent B). The used elution gradient was: from 100 to $90 \% \mathrm{~A}$ in $7 \mathrm{~min}$, from 90 to $80 \% \mathrm{~A}$ in $20 \mathrm{~min}$, from 80 to $60 \% \mathrm{~A}$ in $8 \mathrm{~min}$, from 60 to $35 \% \mathrm{~A}$ in $10 \mathrm{~min}$, isocratic at $35 \% \mathrm{~A}$ for $5 \mathrm{~min}$, from 35 to $0 \% \mathrm{~A}$ in 2 
$149 \mathrm{~min}$, isocratic at $0 \% \mathrm{~A}$ for $3 \mathrm{~min}$, and then from $0 \%$ to $100 \% \mathrm{~A}$ in $5 \mathrm{~min}$. Total run time was $60 \mathrm{~min}$ at a

150 flow rate of $1 \mathrm{~mL} / \mathrm{min}$. All further parameters of the HPLC-DAD-MS ${ }^{\mathrm{n}}$ system were set as previously 151 described at Schweiggert et al. (2009).

152

153 For quantitative betacyanin analyses, extraction and quantification were done following Von Elbe 154 (2001): freeze-dried tissue was pulverised in a ball-mill to a fine powder and then extracted in water using $100 \mathrm{mM}$ MES buffer (2-N-morpholino-ethanesulfonic acid) with pH adjusted to 5.5 using $\mathrm{NaOH}$ and ascorbic acid at $50 \mathrm{mM}$ (Strack et al. 2003). $1.8 \mathrm{ml}$ of extracting solvent was added to $60 \mathrm{mg}$ of tissue powder and first mixed in a vortex for $1 \mathrm{~min}$ and then in a mechanical shaker for 3 hours at $4^{\circ} \mathrm{C}$ in the dark. Samples were then centrifuged for 20 minutes at $15,000 \mathrm{rpm}\left(4{ }^{\circ} \mathrm{C}\right)$. The supernatants were filtered $(0.45 \mu \mathrm{m})$ and $1.2 \mathrm{ml}$ was transferred to a disposable cuvette for spectral absorbance readings. Blanks of $1.2 \mathrm{ml}$ of extracting solvent were recorded every 10 samples to ensure no drift in the calibration of the instrument. Betacyanin absorbance was read at $538 \mathrm{~nm}$ (Strack et al. 2003) on a Cary 3 UV/Vis spectrophotometer (Varian Inc., Palo Alto, CA, USA, spectral bandwidth range 0.2-4 nm; wavelength accuracy $\pm 0.2 \mathrm{~nm})$. Betacyanin concentrations were calculated using $727 \mathrm{~g} \mathrm{~mol}^{-1}$ as the molecular weight and $60 \mathrm{~L} \mathrm{~mol} \mathrm{~cm}^{-1}$ as the molar extinction coefficient (von Elbe 2001) and were expressed on a dry weight basis. Chlorophylls and carotenoids were extracted using cold methanol 166 according to Sims and Gamon (2002). $60 \mathrm{mg}$ of tissue powder was added to $1.8 \mathrm{ml} 100 \%$ cold methanol and mixed thoroughly in a shaker for 3 hours in darkness at $4{ }^{\circ} \mathrm{C}$. Samples were then centrifuged for 15 minutes at $1500 \mathrm{rpm}$ at $4^{\circ} \mathrm{C}$. The supernatant was filtered $(0.45 \mu \mathrm{m})$ and absorbance was measured in the same spectrophotometer as described above, at 470, 652.4 and $665 \mathrm{~nm}$. Chlorophylls and carotenoids were estimated according to equations in Wellburn (1994). 
173 Plant spectral measurements were performed using a handheld spectrometer (JAZ, Ocean Optics 174 Dunedin, FL, USA) to measure reflectance between 400-1200 nm (visible and near-infrared 175 wavelengths) but due to high noise at the end of the spectrum, we only used the bands between $400-800$ $176 \mathrm{~nm}$. The spectrometer has a spectral resolution of $0.35 \mathrm{~nm}$, giving a total of 1139 bands. Reflectance 177 was estimated as a percentage between a maximum, a pure white reference surface, and a minimum 178 reference value obtained by blocking all light from the fiber optic. Measurements were performed 179 between 12:00 and 13:00 on two consecutive cloud-free days. Plant spectral measurements were taken 180 from the north at two angles, $45^{\circ}$ and $90^{\circ}$ as determined by a clinometer. Distance between the sensor 181 and the target was $10 \mathrm{~cm}$.

In order to find an index which best estimated pigment concentration across seasons and species, the Pearson correlation coefficient (r) was calculated between pigment concentration (for betacyanin and chlorophyll) and all possible two-band normalised difference indices of the form:

$$
I_{i j}=\left(\lambda_{i}-\lambda_{j}\right) /\left(\lambda_{i}+\lambda_{j}\right)
$$

where $\lambda$ is the reflectance in the wavelengths $i$ and $j$. As we had spectral measurements with 1139 bands the number of all possible two-band combinations was 1295043 (noting that inverting the $i$ and $j$ in the equation above yields the same correlation with inverted sign). By taking this approach, we are also effectively including some indices which have already been used by researchers for different purposes, for example, the Photochemical Reflectance Index (PRI $\left.{ }_{570}\right)$ (Peñuelas et al. 2011) which has been shown to be sensitive to carotenoid pigments, and which corresponds with $\lambda_{i}$ and $\lambda_{j}$ of 531 and $570 \mathrm{~nm}$. The widely used Normalised Difference Vegetation Index (NDVI) (Gamon and Surfus 1999) uses the reflectance in the near-infrared (NIR) and red bands, but different authors have used different wavelengths for NIR and red and it has also been used with broadband sensors (Table S1). 
197 In order to assess the possible influence of chlorophyll on the correlation between betacyanin and

198 spectral indices, with the aim of identifying the bands most sensitive to betacyanins per se, we fitted the 199 following model following Guerschman et al. (2009):

200

201

$$
C_{\text {betacyanin }}=a_{0}+a_{1} * C_{\text {chlorophyll }}+a_{2} * I_{i j}
$$

(Equation 2)

202

where $C_{\text {betacyanin }}$ and $C_{\text {chlorophyll }}$ refer to the concentration of both pigments in $\mathrm{mg} \mathrm{g}^{-1}$ dry matter, $a_{0}, a_{1}$ and 204 $a_{2}$ are the parameters associated to the linear model and $I_{i j}$ corresponds, as in Equation 1 to all possible two-band indices of the form $I_{i j}=\left(\lambda_{i}-\lambda_{j}\right) /\left(\lambda_{i}+\lambda_{j}\right)$. By finding the values of $i$ and $j$ which maximise the correlation index of the multiple linear model we are effectively identifying a normalised index that best

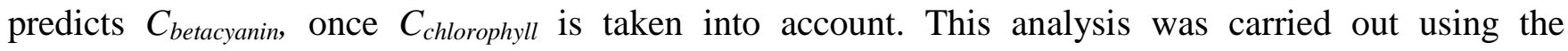
chemically determined chlorophyll concentrations, as well as the spectrally estimated chlorophyll concentrations.

\subsection{Statistical analysis}

Pigment concentrations and spectral values were compared between dates and species using a repeated measures analysis (Littell et al. 1996) which was performed using the Pro-Mixed program from SAS (1999). In all cases we used a significance level of $\mathrm{P}<0.05$.

\section{Results}

\subsection{Identification of Tecticornia sp. betacyanins by HPLC-DAD-MS ${ }^{n}$}

In both $T$. indica and $T$. auriculata, an identical set of compounds with betacyanin-specific absorption properties was detected by HPLC-DAD-MS ${ }^{\mathrm{n}}$ (Fig. 2). Identification of amaranthine (1), isoamaranthine 
221

222 tricolor sample. For the latter, the betalain profile including UV/Vis absorption and mass data was 223 previously reported by Cai et al. (2005). After verifying the previously reported identity of amaranthine 224 (1), isoamaranthine (2), betanin (3), and isobetanin (4) in the Amaranthus sample by HPLC-DAD-MS ${ }^{\mathrm{n}}$ 225 (Table 1), the respective compounds 1-4 were identified in both Tecticornia samples by co226 chromatography and a corresponding comparison of the analytical data reported in table 1 . The profile 227 of water-soluble pigments was highly similar in both Tecticornia sp., containing the betacyanins 228 (Cai et al., 2005). amaranthine (1) and isomaranthine (2) as major as well as betanin (3) and isobetanin (4) as minor constituents (Fig. 2). In addition, the betacyanins detected in Tecticornia were strikingly similar to those found in the Amaranthus sample (Fig.2), being characteristic for several members of the Amaranthaceae

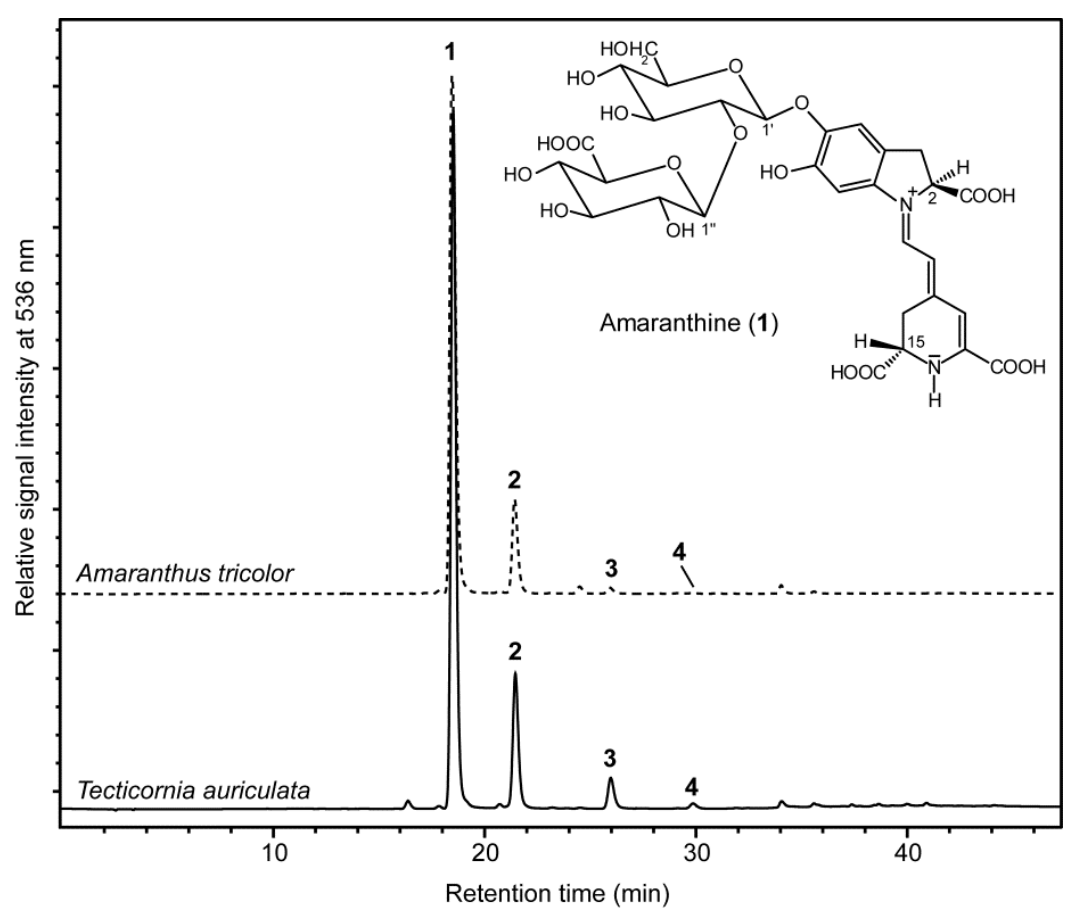

Figure 2. HPLC separation of betacyanins from T. auriculata and A. tricolor leaves. Pigment profiles of T. indica and T. auriculata were identical (T. indica is not shown). Peak assignment, 1: amaranthine, 2: isoamaranthine, 3: betanin, 4: isobetanin (see table 1). 
237 Table 1. HPLC retention times, UV/Vis absorption maxima and mass spectral data of betalains from 238 Tecticornia spp. and Amaranthus caudatus.

\begin{tabular}{|c|c|c|c|c|c|}
\hline $\begin{array}{l}\text { Peak } \\
\text { No. }\end{array}$ & $\begin{array}{c}\text { Compound } \\
\text { identity }\end{array}$ & $\begin{array}{c}\text { HPLC } \\
\text { retention } \\
\text { time } \\
\text { [min] }\end{array}$ & $\begin{array}{c}\mathrm{UV} / \mathrm{Vis} \\
\text { absorption } \\
\text { maximum } \\
{[\mathrm{nm}]}\end{array}$ & $\begin{array}{c}{[\mathrm{M}+\mathrm{H}]^{+}} \\
m / z\end{array}$ & $\begin{array}{c}\text { HPLC-ESI-(+)-MS } \\
m / z \text { (rel. intensity) }\end{array}$ \\
\hline 1 & Amaranthine & 18.3 & 535 & 727 & $\begin{array}{c}\text { [727]: } 389(100), 551 \\
(37)\end{array}$ \\
\hline 2 & Isoamaranthine & 21.4 & 535 & 727 & $\begin{array}{c}\text { [727]: } 389(100), 551 \\
(21)\end{array}$ \\
\hline 3 & Betanin & 26.0 & 535 & 551 & [551]: $389(100)$ \\
\hline 4 & Isobetanin & 29.8 & 535 & 551 & [551]: $389(100)$ \\
\hline
\end{tabular}

\subsection{Pigment dynamics}

241 Betacyanin and chlorophyll concentrations showed strong seasonal dynamics, with opposing trends,

242 whereas carotenoid concentrations were relatively stable. Betacyanin concentrations increased almost 243 nine times in both species (Fig. 3, P<0.001) from the end of the wet summer (March) to the end of the 244 dry winter (August), while plant colour changed from green to red-pinkish (Fig S2). No significant 245 differences in betacyanin concentrations were detected between species ( $\mathrm{P}=0.53$, Fig. 3). For $T$. indica, 246 betacyanin concentration was higher during July and then declined significantly towards the end of 247 August. In T. auriculata betacyanin concentration continued to increase until the end of the dry season, 248 although the differences between July and August were not statistically significant (P=0.22, Fig. 3). 249 Contrary to betacyanin concentrations, chlorophyll concentrations were significantly higher during 
250 March and significantly $(\mathrm{P}<0.05)$ lower during July-August. Carotenoid concentrations declined slowly, 251 showing significantly lower values at the end of the dry season $(\mathrm{P}<0.05$, Fig. 3$)$.

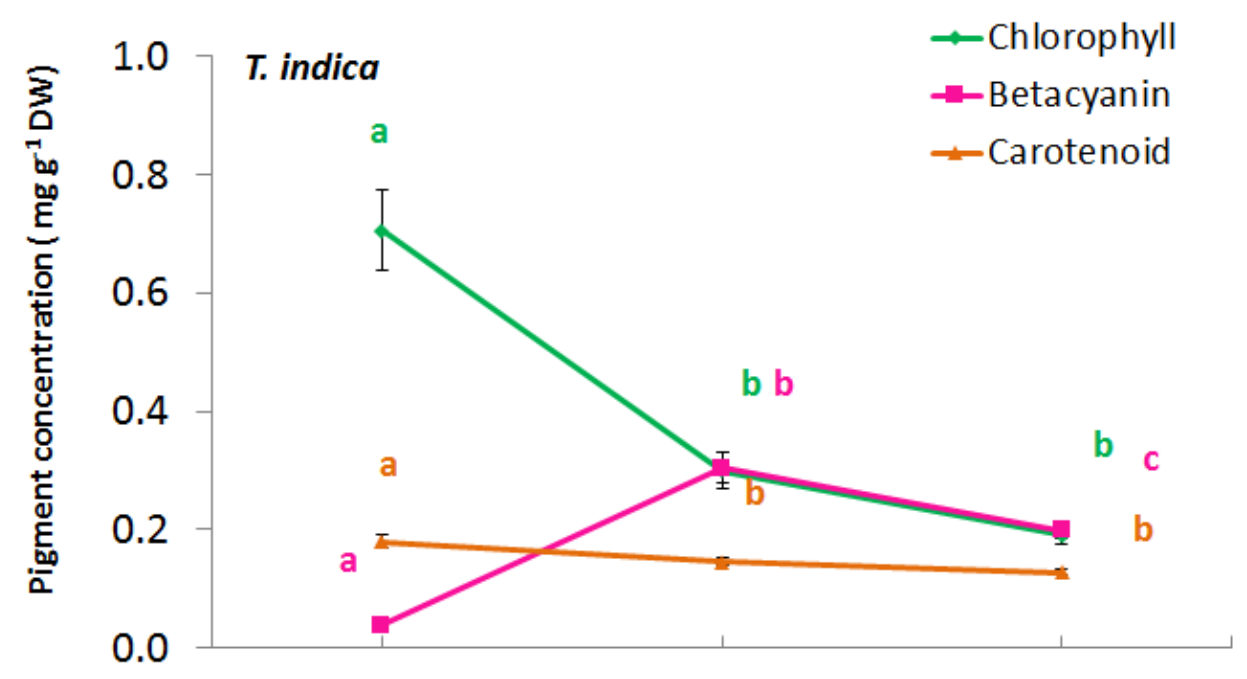

252

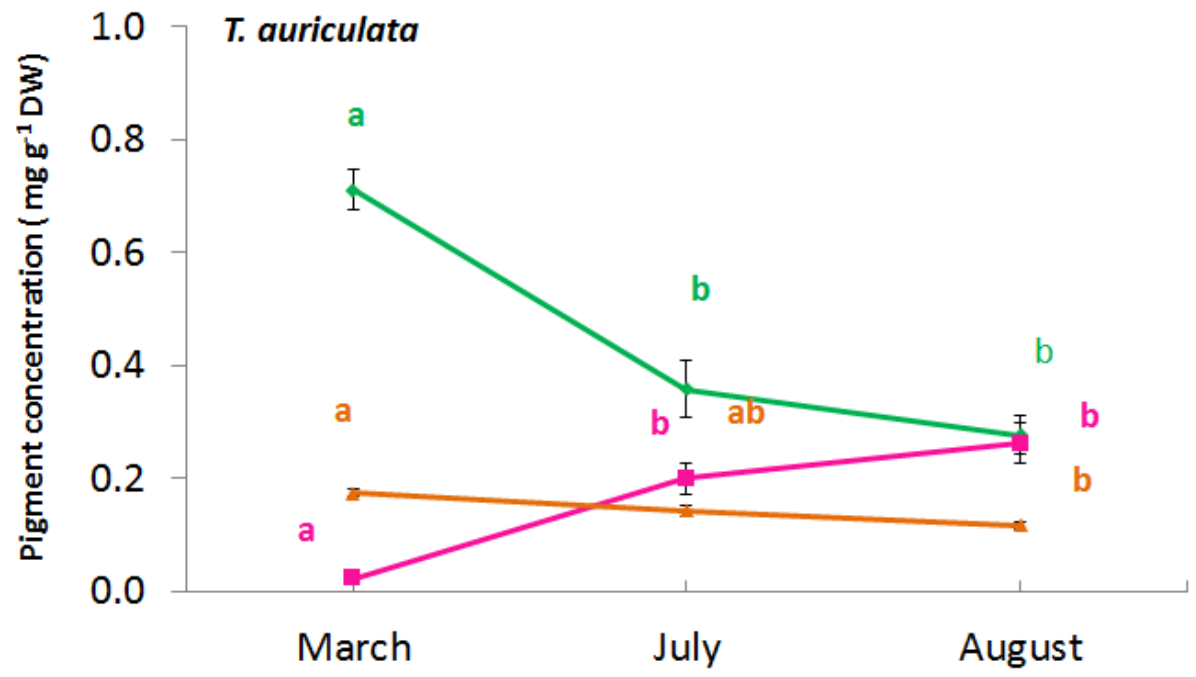

Figure 3: Betacyanin, chlorophyll and carotenoid concentrations for T. auriculata and

T. indica at Fortescue Marsh for March, July and August 2012. Different letters indicate significant differences with time for each individual pigment at $\mathrm{P}=0.05$. Precipitation in the month preceding the sampling was 40.6, 5.0 and $0.0 \mathrm{~mm}$ for the March, July and August samplings, respectively. 


\subsection{Spectral reflectance}

262 Tecticornia reflectance spectra changed seasonally along with the pigments dynamics. As trends were 263 quantitatively and qualitatively similar for the two species, correlations between pigment composition 264 and reflectance spectra were analysed for the combined dataset comprising both species. The best 265 correlation between betacyanin concentration and spectral reflectance was found for the normalised ratio using the bands at 600 and $620 \mathrm{~nm}$ ( $\mathrm{r}=0.805$, Fig. 4), but high correlations were also found for bands in the range 510-550 nm, which indicates a possible influence of the peak absorption of betacyanins at near $540 \mathrm{~nm}$ (Fig. 4). Among the indices most established in literature, the Structure Insensitive Pigment Index (SIPI) (Peñuelas et al. 1995) was the one that best correlated $(r=0.73)$ with betacyanin. In contrast, other indices showed weak or no correlation with betacyanin concentration (Table S1). the bands at 737 and $726 \mathrm{~nm}$, which detects the 'red edge effect' (Sims and Gamon 2002). Also, high correlation values were found between chlorophyll and indices calculated using the bands in the range of 600-620 and 580-600 nm (Fig. 5). The clear opposing patterns of Figures 4 and 5 show that concentrations of betacyanin and chlorophyll were strongly and negatively correlated with each other. 


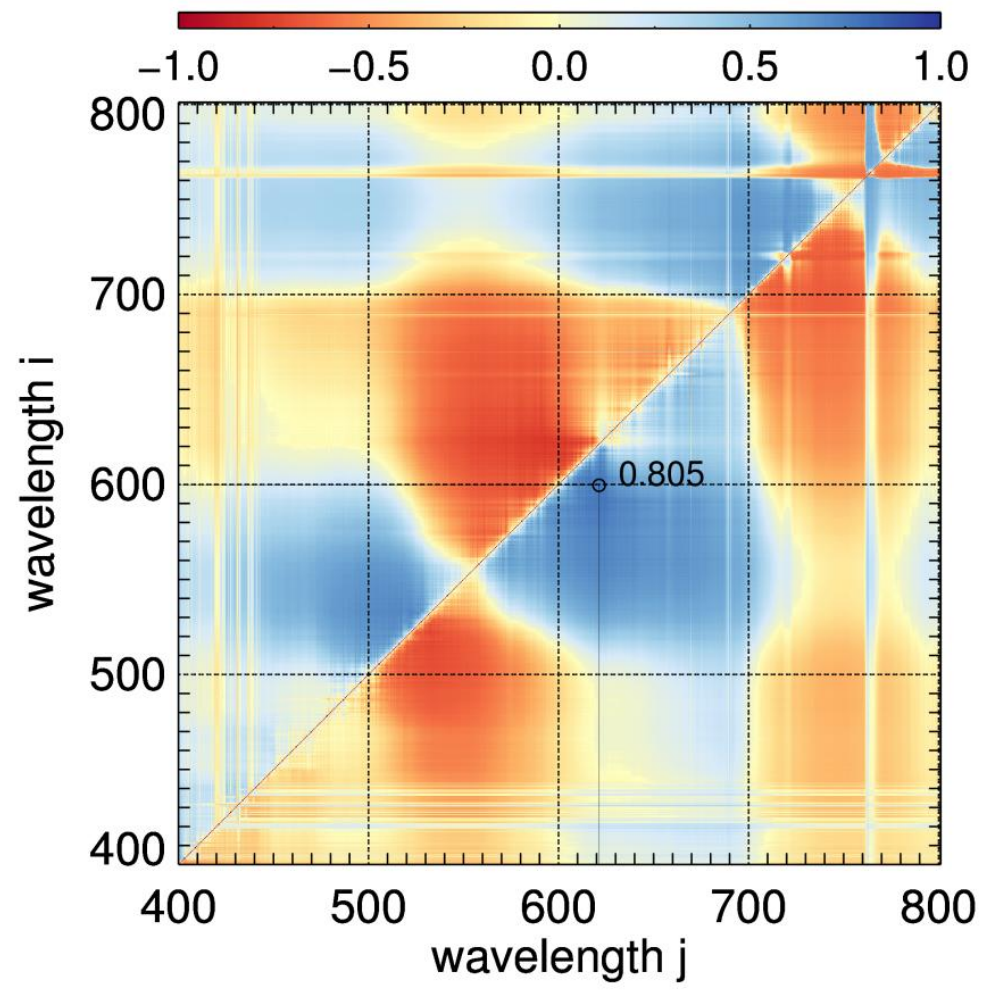

278

279

280

281

282

283

284

285

286

287

Figure 4: Correlation coefficients between betacyanin concentration $\left(\mathrm{mg} \mathrm{g}^{-1} \mathrm{DW}\right)$ and different normalised wavelength ratios for T. auriculata and T. indica. The correlations are based on observations for both species at three different dates in 2012. The difference normalised ratios using 600 and $620 \mathrm{~nm}$ showed the highest correlation with betacyanin concentration $(\mathrm{r}=0.805)$. Note that the correlation array is symmetrical (with inverted sign) along the diagonal axis. 


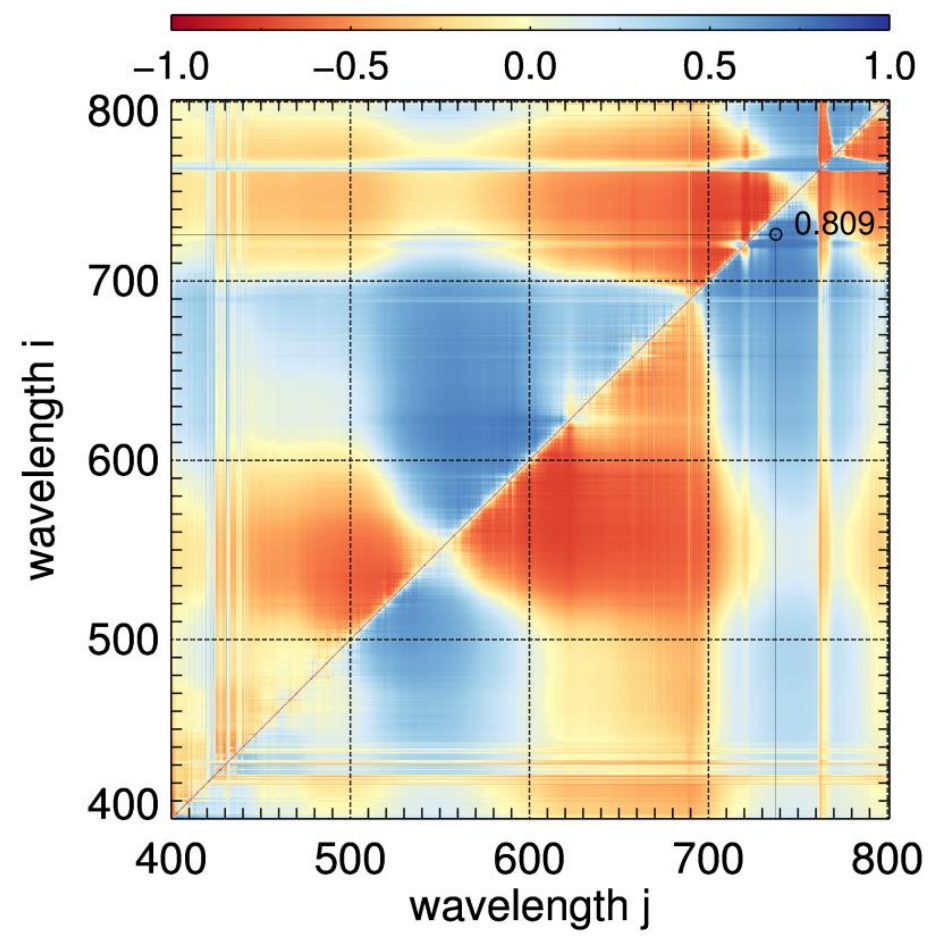

Figure 5: Correlation coefficients between chlorophyll $(\mathrm{a}+\mathrm{b})$ concentration $\left(\mathrm{mg} \mathrm{g}^{-1}\right.$ DW) and different normalised wavelength ratios for T. auriculata and T. indica. The correlations are based on observations for both species at three different dates in 2012. The normalised ratio for 726 and $737 \mathrm{~nm}$ showed the highest correlation with chlorophyll concentration $(r=0.809)$.

295 Given the strong negative correlation between betacyanin and chlorophyll concentrations, it is important 296 to examine if consideration of the chlorophyll concentration can improve the ability of the spectral index 297 to predict betacyanin concentration. Figure 6 shows correlation coefficients for multiple correlations 298 based on Equation 2, using measured chlorophyll concentration and normalised indices of all possible 299 wavelengths. The normalised index using the wavelengths 606 and $621 \mathrm{~nm}$ allowed for a better 300 prediction of betacyanin concentration $(r=0.822)$ than the index that did not consider chlorophyll 
301 concentration (Fig. 4). The parameters $a_{0}, a_{1}$ and $a_{2}$ for this model were $0.309,-0.160$ and -2.373 302 respectively.

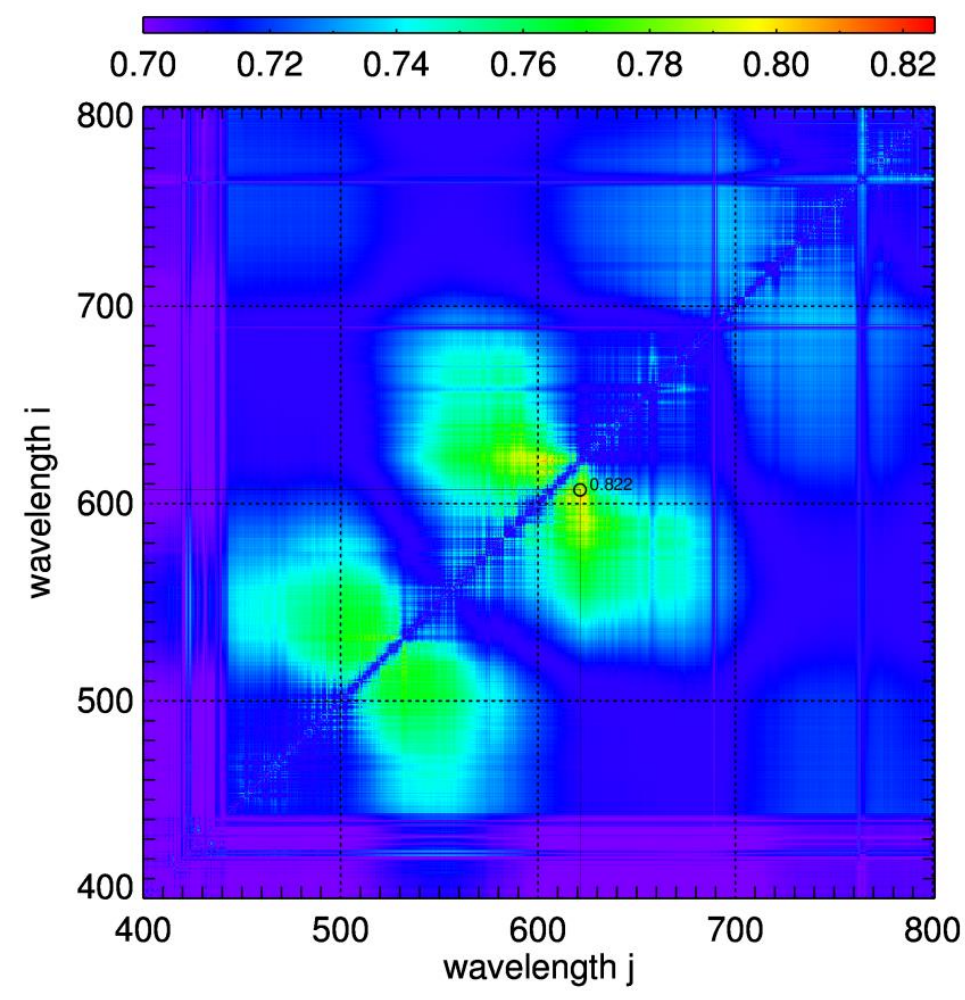

303

Figure 6: Correlation coefficients for the multiple correlation of betacyanin concentration ( $\left.\mathrm{mg} \mathrm{g}^{-1} \mathrm{DM}\right)$ with different normalised wavelength ratios $(\mathrm{nm})$ and measured chlorophyll concentrations. The analysis is based on combined data for $T$. auriculata and T. indica for three dates in 2012.

309 The regression between the observed and the predicted betacyanin concentration using the normalized

310 ratio 606/621 and measured chlorophyll concentration showed a good fit $\left(r^{2}\right.$ 0.67, Fig 7$)$, suggesting a

311 potential and reliable use of this method to estimate this pigment via remote sensing. 


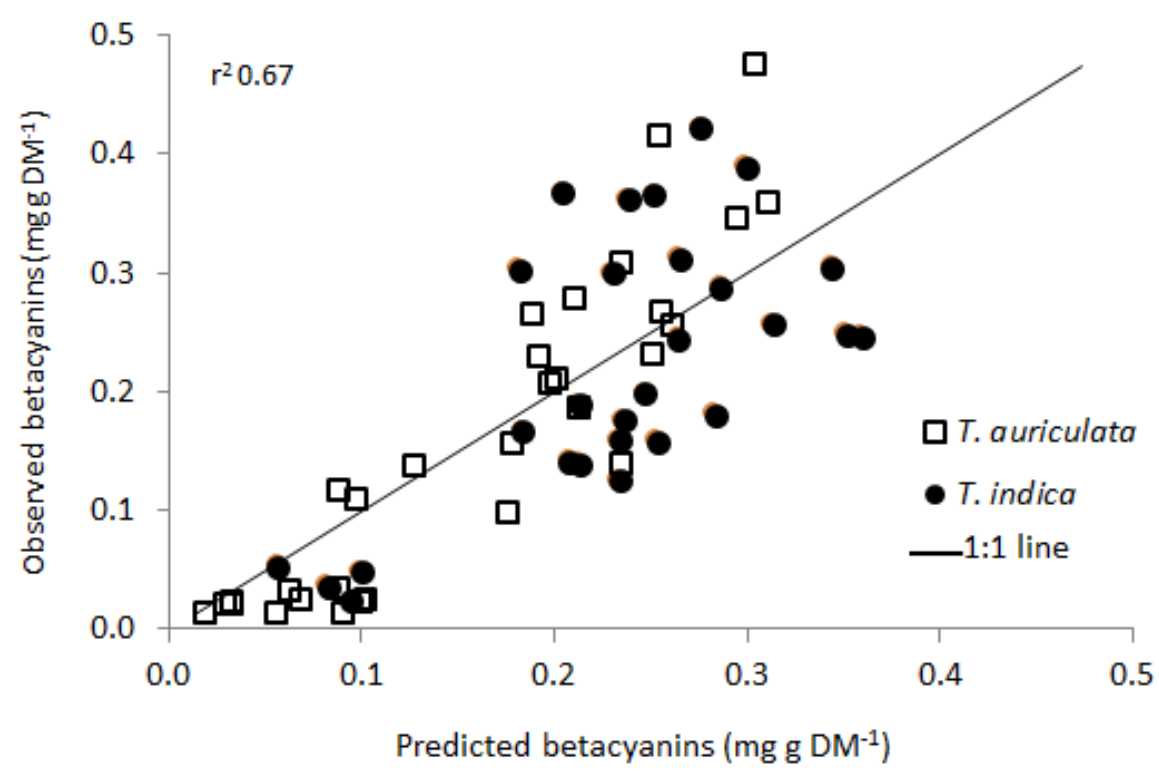

Figure 7: Observed versus predicted betacyanin concentration for $T$. indica and $T$. auriculata for March, July and August 2012. Predicted values obtained according the model in Equation 2 using the normalised index of wavelengths 606 and $621 \mathrm{~nm}$.

317

318 Finally, having established that betacyanin concentrations could be estimated with the new normalised

319 difference index, and that taking into account the (chemically) measured chlorophyll concentrations improved the accuracy of the betacyanin estimates, we then performed a final test to determine if the same improved accuracy could be achieved using spectrally estimated chlorophyll concentrations. Chlorophyll was estimated using the wavelengths 726 and $737 \mathrm{~nm}$ (Fig. 5) and then used to fit Equation (Fig. S2), almost as good as in the model using measured chlorophyll concentrations. Thus, both pigments, chlorophyll and betacyanin, can be simultaneously spectrally detected using the models and the wavelengths presented here. 
329

330

331

332

333

334

335

336

337

338

339

340

341

342

343

344

345

346

347

348

349

350

351

352

Variations in pigmentation in leaves and other tissues have been widely studied in relation with plant phenological and physiological changes (Demmig-Adams 1990, Chalker-Scott 1999, Lee 2002). However, despite its high potential value, the detection of these changes by spectrometry is still underdeveloped, especially for pigments other than chlorophylls. In this study, we showed that seasonal pigment changes of two stem-succulent halophytic shrubs (Tecticornia species), specifically the redpinkish betacyanins, can be detected using remote sensing.

Seasonal changes in betacyanin concentrations were observed in the two Tecticornia species, with levels increasing from the wet season to the dry season. Changes in betacyanins have also been described for other species in the Amaranthaceae with an increase in levels under stress. As examples, in Suaeda salsa (Zhao et al. 2010), Suaeda japonica (Hayakawa and Agarie 2010) and Sarcocornia fructicosa (Duarte et al. 2013) betacyanin synthesis increased after increased light intensity, lower temperatures and during drought. In these species betacyanins appear to be the major stress-related pigment, more than anthocyanins or carotenoids. Chlorophylls in the two Tecticornia species, in contrast with the betacyanins, were highest during the wet-season and decreased during the dry season. However, carotenoids did not show substantial changes over time. Given the potential for a protective role of betacyanins under stress conditions (Stintzing and Carle 2004), a role that also carotenoids have (Young 1991), it is likely that betacyanins play an important function in Tecticornia species.

The changes observed in the two Tecticornia species due to betacyanins colouration was accurately detected by using a linear model considering the total chlorophyll concentration (which was also spectrally estimated using the normalized ratio of reflectance at wavelengths $726 / 737 \mathrm{~nm}$ proposed in this study), and a normalized ratio for betacyanins using the reflectance at wavelengths $621 / 606 \mathrm{~nm}$ as also proposed here. Gamon and Surfus (1999), who have explored many spectral indices related to 
353

354

355

356

357

358

359

360

361

362

363

364

365

366

367

368

369

370

371

372

373

374

375

376

377

chlorophylls and anthocyanins, have concluded that compared to other indices, the two-bands reflectance ratio approach has the advantage of reducing the multidimensional spectrum to a few values. However, although indices such as PRI or ARI have demonstrated strong correlation with changes in carotenoids and anthocyanins, respectively, in other species (Stylinski et al. 2002, Gitelson et al. 2006), these indices showed a weak correlation with betacyanins in the two Tecticornia species (Table S1). Due to the prominence of betacyanins in Tecticornia shoot tissues, the new proposed index detects seasonal pigment changes more accurately than those other two existing indices.

Although our results relate to measurements at plant scale, the dominance of Tecticornia species in the outer areas of Fortescue Marsh (> 90\%) plus the strong seasonality observed in their pigments makes detecting these changes at larger scales plausible. A preliminary study done at plot level and using the same technique (and device) confirmed the significant increase of the normalised index using 606/621 $\mathrm{nm}$ wavelengths for this vegetation towards the end of the dry season, corresponding with observed changes in pigments in these Tecticornia plants (V. Marchesini, unpublished data). We recommend further studies at larger scales, including the evaluation of the normalised index $606 / 621 \mathrm{~nm}$ that we propose for assessment of betacyanins, using airborne or satellite hyperspectral remote sensors.

Tecticornia species cover a large proportion of saline ecosystems of Australia (Wilson 1980) and might be suitable for revegetation/stabilisation of degraded mining or saline areas (Thompson and Thompson 2004). The spectral detection of vegetation condition by observing changes in betacyanins, as well as chlorophylls, is a promising method to monitor seasonal changes and might also enable detection of other, non-seasonal changes including from possible impacts on Tecticornia communities. The new spectral index presented in this study offers an alternative for other indices in vegetation dominated by species with betacyanin pigments. 


\section{5. Acknowledgments}

380 This study was funded by the ARC Linkage and Fortescue Metals Group Ltd (Grant LP0882350). The 381 authors thank Louis Moir-Barnetson, Daniel Huxtable, Antony Taggart, Timothy Higgott and Craig 382 Douglas for information provided and for field assistance, Greg Cawthray for advice on pigment 383 determinations, and Ms. Elena Sperl for her assistance regarding the HPLC analyses. V. Marchesini was 384 supported by an Australia Endeavour Award Postdoctoral Fellowship. 


\section{References}

401

Archetti, M. 2009. Classification of hypotheses on the evolution of autumn colours. Oikos 118:328-333.

402

Brockington, S. F., R. H. Walker, B. J. Glover, P. S. Soltis, and D. E. Soltis. 2011. Complex pigment

403 evolution in the Caryophyllales. New Phytologist 190:854-864.

404

Cai, Y., Sun, M., \& Corke, H. (2005). HPLC Characterization of betalains from plants in the Amaranthaceae. Journal of Chromatographic Science, 43: 454-460 Photochemistry and Photobiology 70:1-9.

Datson, B., editor. 2002. Samphires in Western Australia: A field guide to Chenopodiaceae tribe Salicornieae, Perth, Australia. zeaxanthin. Biochimica et Biophysica Acta - Bioenergetics 1020:1-24.

Demmig-Adams, B. 1990. Carotenoids and photoprotection in plants: A role for the xanthophyll

Duarte, B., D. Santos, J. C. Marques, and I. Caçador. 2013. Ecophysiological adaptations of two halophytes to salt stress: Photosynthesis, PS II photochemistry and anti-oxidant feedback -

Gamon, J. A., J. Peñuelas, and C. B. Filed. 1992. A narrow-waveband spectral index that tracks diurnal changes in photosynthetic efficiency. Remote Sensing of the Environment 41:35-44.

420 Gamon, J. A. and J. S. Surfus. 1999. Assessing leaf pigment content and activity with a reflectometer. New Phytologist 143:105- 117. 
422 Gitelson, A. A., O. B. Chivkunova, and M. N. Merzlyak. 2009. Nondestructive estimation of

423

424

425

426

427

428

429

430

431

432

433

434

435

436

437

438

439

440

441

442

443

444

445 anthocyanins and chlorophylls in anthocyanic leaves. American Journal of Botany 96:18611868.

Gitelson, A. A., G. P. Keydan, and M. N. Merzlyak. 2006. Three-band model for noninvasive estimation of chlorophyll, carotenoids, and anthocyanin contents in higher plant leaves. Geophysical Research Letters 33:L11402.

Guerschman, J. P., M. J. Hill, L. J. Renzullo, D. J. Barrett, A. S. Marks, and E. J. Botha. 2009.

Estimating fractional cover of photosynthetic vegetation, non-photosynthetic vegetation and bare soil in the Australian tropical savanna region upscaling the EO-1 Hyperion and MODIS sensors. Remote Sensing of the Environment 113:18-18.

Hayakawa, K. and S. Agarie. 2010. Physiological roles of betacyanin in a halophyte, Suaeda japonica Makino. Plant Production Science 13:351-359.

Ibdah, M., A. Krins, H. K. Seidlitz, W. Heller, D. Strack, and T. Vogt. 2002. Spectral dependence of flavonol and betacyanin accumulation in Mesembryanthemum crystallinum under enhanced ultraviolet radiation. Plant, Cell \& Environment 25:1145-1154.

Kelly, M., K. A. Tuxen, and D. Stralberg. 2011. Mapping changes to vegetation pattern in a restoring wetland: finding pattern metrics that are consistent across spatial scale and time. Ecological Indicators 11:263-273.

Kerr, J.T., \& Ostrovsky, M. (2003). From space to species: ecological applications for remote sensing. Trends in Ecology \& Evolution 18: 299-305

Lee, D.W. (2002). Anthocyanins in autumn leaf senescence. Advances in Botanical Research. 37:147165. Academic Press

Littell, R. C., G. A. Milliken, W. W. Stroup, and R. D. Wolfinger. 1996. SAS System for Mixed Models. SAS Institute Inc, Cary, NC. 
446 Moir-Barnetson, L. (2015). Ecophysiological responses to changes in salinity and water availability in stem-succulent halophytes (Tecticornia spp.) from an ephemeral salt lake. PhD Thesis. Plant Biology. The University of Western Australia

Ollinger, S. V. 2011. Sources of variability in canopy reflectance and the convergent properties of plants. New Phytologist 189:375-394.

Peñuelas, J., F. Baret, and I. Filella. 1995. Semi-empirical indices to assess carotenoids/chlorophyll a ratio from leaf spectral reflectance. Photosynthetica 31:221-230.

Peñuelas, J., M. F. Garbulsky, and I. Filella. 2011. Photochemical reflectance index (PRI) and remote sensing of plant CO2 uptake. New Phytologist 191:596-599.

Rabhi, M., A. Castagna, D. Remorini, C. Scattino, A. Smaoui, A. Ranieri, and C. Abdelly. 2012. Photosynthetic responses to salinity in two obligate halophytes: Sesuvium portulacastrum and Tecticornia indica. South African Journal of Botany 79:39-47.

SAS. 1999. Comparing the SAS GLM and MIXED procedures for repeated measures. R. Wolfinger and M. Chang, editors. SAS Institute Inc, Cary, NC.

Schweiggert, R., Villalobos-Gutierrez, M., Esquivel, P., \& Carle, R. (2009). Development and optimization of low temperature enzyme-assisted liquefaction for the production of colouring foodstuff from purple pitaya (Hylocereus sp. [Weber] Britton \& Rose). European Food Research and Technology, 230: 269-280

Shepherd, K. A. and S. J. Van Leeuwen. 2010. Tecticornia globulifera and T. medusa (subfamily Salicornioideae: Chenopodiaceae), two new priority samphires from the Fortescue Marsh in the Pilbara region of Western Australia. Telopea 13:349-358.

Shepherd, K. A. and P. G. Wilson. 2007. Incorporation of the Australian genera Halosarcia, Pachycornia, Sclerostegia and Tegicornia into Tecticornia (Salicornioideae, Chenopodiaceae). Australian Systematic Botany 20 319-331. 
470 Shuman, C. S. and R. F. Ambrose. 2003. A Comparison of remote sensing and ground-based nethods for monitoring wetland restoration success. Restoration Ecology 11:325-333.

472

473

474

475

476

477

478

479

480

481

482

483

484

485

486

487

488

489

490

491

492

493

Sims, D. A. and J. A. Gamon. 2002. Relationships between leaf pigment content and spectral reflectance across a wide range of species, leaf structures and developmental stages. Remote Sensing of Environment 81:337- 354.

Skrzypek, G., S. Dogramaci, and P. F. Grierson. 2013. Geochemical and hydrological processes controlling groundwater salinity of a large inland wetland of northwest Australia. Chemical Geology 357:164-177.

Stintzing, F. C. and R. Carle. 2004. Functional properties of anthocyanins and betalains in plants, food, and in human nutrition. Trends in Food Science \& Technology 15:19-38.

Strack, D., T. Vogt, and W. Schliemann. 2003. Recent advances in betalain research. Phytochemistry 62:247-269.

Stylinski, C., J. Gamon, and W. Oechel. 2002. Seasonal patterns of reflectance indices, carotenoid pigments and photosynthesis of evergreen chaparral species. Oecologia 131:366-374.

Thompson, S. A. and G. G. Thompson. 2004. Adequacy of rehabilitation monitoring practices in the Western Australian mining industry. Ecological Management \& Restoration 5:30-33.

Timms, B. 2005. Salt Lakes in Australia: Present problems and prognosis for the future. Hydrobiologia 552:1-15.

Ustin, S. L., A. A. Gitelson, S. Jacquemoud, M. Schaepman, G. P. Asner, J. A. Gamon, and P. ZarcoTejada. 2009. Retrieval of foliar information about plant pigment systems from high resolution spectroscopy. Remote Sensing of Environment 113:67-77.

Ustin, S. L., D. A. Roberts, J. A. Gamon, G. P. Asner, and R. O. Green. 2004. Using imaging spectroscopy to study ecosystem processes and properties. BioScience 54:523-543.

von Elbe, J. H. 2001. Betalains. Current protocols in food analytical chemistry. John Wiley \& Sons, Inc. 
494 Wellburn, A. R. 1994. The Spectral determination of chlorophylls a and b, as well as total carotenoids, 495 using various solvents with spectrophotometers of different resolution. Journal of Plant $496 \quad$ Physiology 144:307-313.

497 Wilson, P. G. 1980. A revision of the Australian species of Salicornia (Chenopodiaceae). Nuytsia 3:3$498 \quad 154$.

499 Young, A. J. 1991. The photoprotective role of carotenoids in higher plants. Physiologia Plantarum $500 \quad 83: 702-708$.

501 Zhao, S.-Z., H.-Z. Sun, M. Chen, and B.-S. Wang. 2010. Light-regulated betacyanin accumulation in 502 euhalophyte Suaeda salsa calli. Plant Cell, Tissue and Organ Culture (PCTOC) 102:99-107. 503 Zomer, R. J., A. Trabucco, and S. L. Ustin. 2009. Building spectral libraries for wetlands land cover 504 classification and hyperspectral remote sensing. Journal of Environmental Management 90: $505 \quad 2170-2177$.

506

507 